\title{
Is a Plasmodium lactate dehydrogenase (pLDH) enzyme-linked immunosorbent (ELISA)-based assay a valid tool for detecting risky malaria blood donations in Africa?
}

Pascal S Atchade ${ }^{1,2+}$, Cécile Doderer-Lang ${ }^{1 \dagger}$, Nicodème Chabi ${ }^{2}$, Sylvie Perrotey ${ }^{1}$, Tamer Abdelrahman $^{3}$, Casimir D Akpovi ${ }^{2}$, Ludovic Anani ${ }^{4}$, André Bigot ${ }^{4}$, Ambaliou Sanni ${ }^{2 \dagger}$ and Ermanno Candolfi ${ }^{*+}$

\begin{abstract}
Background: Malaria is a leading cause of mortality in southern Benin. The main causative agent, Plasmodium falciparum, poses a threat on critical transfusions in pregnant women and children. This study's objective was to compare the performance of different malaria screening methods in blood donors in southern Benin, a malaria-endemic country.

Methods: Blood from 2,515 voluntary blood donors in Benin was collected over a period of 10 months in ethylenediaminetetraacetic acid (EDTA) tubes, which were then classified according to extraction time: long rainy season, short dry season, short rainy season, and long dry season. Microscopic examination was used to count parasites. Parasite density (PD) was expressed as the number of parasites per $\mu \mathrm{L}$ of blood. Pan Plasmodium pLDH detection was assessed by an ELISA-malaria antigen test. Using crude soluble P. falciparum antigens, an ELISA-malaria antibody test detected anti-Plasmodium antibodies.
\end{abstract}

Results: Among the 2,515 blood donors (2,025 males and 488 females) screened, the rate of asymptomatic Plasmodium carriage was 295/2,515 (11.72\%, 95\% Cl: 10.5-13.1\%). Males had a higher infection rate (12.4\%) than did females (8.8\%). Parasite density was very low: between seven and100 parasites per $\mu \mathrm{L}$ of blood was reported in $80 \%$ of donors with parasitaemia. Three Plasmodium species were diagnosed: P. falciparum in 280/295 patients (95.0\%), Plasmodium malariae in 14/295 (5.0\%), and Plasmodium ovale in 1/295 (0.34\%). Malaria prevalence in donors was higher during the rainy seasons (13.7\%) compared with the dry seasons (9.9\%). The use of a highly sensitive assay enabled pan Plasmodium pLDH detection in 966/2,515 (38.4\%, 95\% Cl: 36.5\%-40.3\%). Malaria antibody prevalence was 1,859/2,515 (73.9\%, 95\% Cl: 72.16-75.6\%). Donors' antigenaemia and antibody levels varied significantly $(P<0.05)$ over the course of the four seasons. The highest antigenaemia rate 323/630 (51.3\%), was observed during the short rainy season, while the highest antibody prevalence, 751/886 (84.7\%), was recorded during the long dry season.

Conclusion: Blood donations infected with Plasmodium can transmit malaria to donation recipients. Malaria diagnostic methods are currently available, but the feasibility criteria for mass screening in endemic areas become preponderant. Detection of the pLDH antigen seems to be an adequate screening tool in endemic areas, for this antigen indicates parasite presence. Routine screening of all donated blood would prevent infected blood donations and reduce P. falciparum transmission in critical patients, such as children and pregnant women. This tool would also decrease medical prophylaxis in donation recipients and contribute to lower Plasmodium resistance.

Keywords: Malaria, Transfusion, Plasmodium falciparum, pLDH, Antibodies

\footnotetext{
*Correspondence: candolfi@unistra.fr

${ }^{\dagger}$ Equal contributors

'Institut de Parasitologie et de Pathologie Tropicale (IPPTS) - Fédération de Médecine Translationnelle, Université de Strasbourg, Strasbourg, France

Full list of author information is available at the end of the article
} 


\section{Background}

Transfusion-induced malaria was first reported in 1911 [1], and it is well-established that all five human malaria parasites (Plasmodium falciparum, Plasmodium malariae, Plasmodium vivax, Plasmodium ovale, and Plasmodium knowlesi) may be transmitted via blood transfusion [2]. Blood is mainly used in the emergency management of patients with life-threatening anaemia accompanied by severe malaria and malnutrition [3]. Blood transmission of malaria is a potentially serious complication that poses a continuous risk for blood banks, as the recipient's malaria diagnosis is not expected, and is thus often missed $[3,4]$. As malaria parasites can survive in red blood cells at refrigerator temperature $\left(2-4^{\circ} \mathrm{C}\right)$ for days or weeks [5], this would require the exclusion of all blood donors with a potential risk [6,7]. Transfusion-transmitted malaria (TTM) is an important public health problem that affects most parts of the world. Over the past decade, TTM has been reported in low endemic countries, such as France [8], Brazil [9], the USA [10], and the UK [11]. The situation is more alarming in highly endemic areas, such as subSaharan Africa, where Plasmodium prevalence among blood donors may reach record levels of $51.50 \%[12,13]$ using a technique less sensitive than microscopy [14].

In Benin, as in other tropical developing countries, the high demand for blood donations due to increased road accidents, pregnancy-related haemorrhages and child anaemia enhances the risk of TTM. Benin's humid tropical climate, which has two rainy and two dry seasons, favours malaria transmission over the course of eight months, with 58 infectious mosquito bites per man per year [15]. The most effective malaria vector, Anopheles gambiae, is the most widespread and difficult to control [16]. The majority of malaria-related deaths in Benin is caused by the lethal P. falciparum. In Benin, 37\% of adult health facility visits and $41 \%$ of those by children under five years old are malaria-related. Malaria is the leading disease affecting communities, with pregnant women and children under five years old being the most vulnerable. The incidence of uncomplicated and severe malaria was 139 per 1,000 inhabitants in 2006 [17]. Selfmedication for malaria prevention is likely to complicate these figures: although widespread in Benin, it has never been objectively assessed. TTM is not considered a priority in malaria-endemic countries, but malaria prevalence is nonetheless decreasing thanks to the Roll Back Malaria programme [18]. The significant risk of Plasmodium transfusion is expected to increase over the next few years in unprotected patients, such as pregnant women or children. Blood transfusion is the third transmission path of Plasmodium, and there is still no consensus on TTM preventative measures to be taken in tropical settings [19]. Malaria symptoms may be subtle or non-existent in many blood donors carrying
Plasmodium [7], so screening questionnaires have limitations [20]. Moreover, routine malaria diagnosis is made by microscopic visualization of parasites on thick and thin smears, but this method is inadequate for examining a large volume of samples $[14,20]$. Nevertheless, microscopy is still commonly used in studies investigating malaria prevalence in blood donors [13,21-23]. The use of malaria antibody detection, as in non-endemic countries, is inadequate due the frequent high seroprevalence in endemic countries. As previously suggested, malaria antigen detection may be a feasible solution $[7,19]$. Therefore, this study's objective was to assess the performance of a commercially available pLDH antigen detection ELISA-based assay compared to microscopy in 2,515 voluntary blood donors who were asymptomatic for malaria. Malaria antibody prevalence was also assessed.

\section{Methods}

\section{Study sites and ethical consideration}

Benin is characterized by tropical rainforest vegetation, Sudanese climate type with an annual rainfall of about $1,600 \mathrm{~mm}$, and an atmospheric temperature of $32^{\circ} \mathrm{C}$. There are four distinct seasons: two rainy and two dry. This descriptive, transversal study was conducted in Benin over ten consecutive months in six departments, which grouped into three pairs of Departmental Blood Transfusion Centres (DBTC): Atlantique-Littoral,OuemePlateau, and Mono-Couffo. They serve as referral centres for south Benin blood transfusion services. This study was approved by the Direction of Benin National Blood Transfusion Agency and the Research Ethics Committee of the Republic of Benin. Approval was granted under the following conditions: donor anonymity must be maintained, good laboratory practice quality control must be ensured, and every finding must be treated with utmost confidentiality and used only for this research purpose. Biological analyses of the samples were performed in the biochemistry and molecular biology laboratory in Cotonou (Benin) and the Institut de Parasitologie et de Pathologie Tropicale de Strasbourg (IPPTS) (France).

\section{Study population}

Individuals considered healthy who visited the three DBTCs for blood donation between May 2009 and March 2010 were considered eligible for the study. A total of 2,515 donors and regular voluntary blood donors were enrolled over a period of ten months, which was divided into a long rainy season (LRS) from May to July, a short dry season (SDS) from August to September, a short rainy season (SRS) from October to November, and a long dry season (LDS) from December to March. Donors were selected according to medical screening criteria: no fever, no clinical evidence of progressive disease, weight above $50 \mathrm{~kg}$, age between 18 and 60 years 
old, or 65 years old for regular donors. Last blood donation had to have occurred within the previous four months for women and three months for men. A more systematic questionnaire was introduced providing the full name, age, sex, address, and telephone of each individual. Malaria-related questions were also included: date and confirmation via clinical manifestation of malaria episodes that occurred during the year, subsequent treatment and prevention measures taken against malaria. Each study participant signed an informed consent. Overall, $7 \mathrm{~mL}$ of blood were collected in EDTA tubes, with $1 \mathrm{~mL}$ transferred into a microtube and the remaining $6 \mathrm{~mL}$ centrifuged at $4^{\circ} \mathrm{C}$, plasma being collected into a new microtube. The tubes were stored at $-20^{\circ} \mathrm{C}$ and transported to the IPPTS laboratory in solid carbon dioxide for antibody and pLDH antigen detection.

\section{Parasitological examination}

A thick and a thin smear were performed on slides for each Benin blood donor. The thick film was dehaemoglobinized using distilled water at $\mathrm{pH}$ 7.2, and then thick and thin blood films were stained using a May-GrünwaldGiemsa (MGG) stain. The microscopic reading and parasite density calculation were performed by two laboratory technicians, and in the case of uncertainty, also by a third one. Parasite count was made on the basis of 500 parasites per 1,000 leukocytes. The reading was stopped after 500 parasites were counted, even though the 1,000 figures of leukocytes were not reached. Parasite density (PD) was expressed as the number of parasites per $\mu \mathrm{L}$ of blood.

\section{pLDH antigen detection}

pLDH is a glycolytic pathway enzyme secreted by the different Plasmodium species, but it possesses speciesspecific isomers [24]. The pLDH enzyme disappears within 24 hours of effective malaria treatment [25]. Therefore, the pLDH antigen is considered a specific marker for the presence of viable Plasmodium in blood, and is used for screening in malaria-endemic countries. The pLDH antigen detection was performed by a sandwich enzyme-linked immunosorbent assay (ELISA), notably an ELISA-malaria antigen test (apDianv, Belgium) that detects $\mathrm{pLDH}$ via immunocapture. The apDia Antigen ELISA is an in vitro diagnostic immunoassay (IVD) for the qualitative determination of Plasmodium spp. LDH in blood samples. The apDia Malaria antigen test can be used in order to detect the malaria pLDH antigen of any of the four species in blood samples. The test was performed according to manufacturer recommendations: pour $100 \mu \mathrm{L}$ of ready-to-use lysing buffer into each well; add $50 \mu \mathrm{L}$ of reconstituted positive control to one well and $50 \mu \mathrm{L}$ of negative control to triplicate well; add 50 $\mu \mathrm{L}$ of homogenized fresh whole blood sample into corresponding well; incubate for $60 \mathrm{~min}$ at $37^{\circ} \mathrm{C}$ under continuous gentle shaking conditions; drain the wells via aspiration, and fill them completely with $350 \mu \mathrm{L}$ of washing solution; allow the wells to soak for $1 \mathrm{~min}$ before washing again five times; pour $100 \mu \mathrm{L}$ of conjugate 1 solution into each well, and incubate the plate for 30 min at $37^{\circ} \mathrm{C}$; wash the wells five times and pour $100 \mu \mathrm{L}$ of conjugate 2 into each wells. Incubate the plate for 15 min then wash the wells five times and pour $100 \mu \mathrm{L}$ of chromogenic solution into each well; incubate the plate for $15 \mathrm{~min}$ at $37^{\circ} \mathrm{C}$; add $50 \mu \mathrm{L}$ of stopping solution to all wells and read absorbance of each well at $450 \mathrm{~nm}$ with reference wavelength of $620 \mathrm{~nm}$ within $15 \mathrm{~min}$. Test results were interpreted as follows: the optical densities of positive control (ODpos) must be $>0.500$ and the average OD of negative control (ODneg) $<0.100$. The ODneg was used to calculate the cut-off by multiplying its average value by three. The antigen index (Ag Index) of each sample was calculated by dividing the OD value of the sample by the cut-off value. A sample was considered positive if the $\mathrm{Ag}$ Index was $\geq 1.0$, indicating that the sample contained viable parasites. A sample was considered negative if the $\mathrm{Ag}$ Index was $\leq 0.8$, indicating that there were no viable parasites in the blood, or that there was no Plasmodium multiplication because of antimalarial drug intake. A sample was considered inconclusive if the Ag Index was between 0.8 and 1.

\section{Measurement of sensitivity, specificity and detectability of the ELISA-based pLDH detection assay Samples from malaria patients for sensitivity calculation}

The whole blood biobank collected from 266 malaria patients who had returned from endemic countries, positively diagnosed and referred by Strasbourg University hospital was tested. Among them, 239 were positive for P. falciparum, 19 for $P$. ovale, six for $P$. malariae, and two exhibited a mixed infection ( $P$. ovale and $P$. falciparum). This panel was used to assess the sensitivity of the ELISA antigen detection test compared to microscopy.

\section{Samples from blood donors for specificity calculation}

Blood donor samples were collected at the French Etablissement Français du Sang d'Alsace (EFS Alsace). Based on a medical questionnaire, donors were classified as "malaria-risk blood donors" $(\mathrm{n}=1,771)$ if they had travelled to an endemic area in the previous four months, or "not-exposed-to-malaria blood donors" $(\mathrm{n}=1,781)$ if they had not travelled to an endemic area in the previous three years.

\section{Cultured parasites}

The 3D7 strain of $P$. falciparum was maintained in vitro using an adapted candle jar method for continuous culture [26]. Parasites were cultured in normal O-positive 
red blood cells (RBCs) from healthy donors (EFS Alsace) and malaria culture medium (MCM, pH 7.4) composed of Roswell Park Memorial Institute medium (RPMI) 1640 supplemented with $2 \mathrm{mM}$ L-glutamine, $10 \mathrm{mM}$ Hepes (Gibco, Invitrogen, Cergy Pontoise, France), $1 \mu \mathrm{g} /$ $\mathrm{ml}$ hypoxanthine, $0.11 \mathrm{mg} / \mathrm{ml} \mathrm{Na}$ pyruvate, $0.02 \mathrm{mg} / \mathrm{ml}$ gentamycin, and $10 \%(\mathrm{~V} / \mathrm{V})$ alpha calf serum (Perbio Science, Brebières, France). The culture was diluted at a parasitaemia and haematocrit of $1 \%$, and the medium was changed every 48 hours.

\section{Determination of parasite detectability threshold using microscopy and pLDH Elisa test}

Culture with $65 \%$ ring-stage parasites and 35\% schizonts was used. Infected RBCs from the continuous culture at $1 \%$ parasitaemia determined on thin blood film by microscopy were serially diluted in uninfected RBCs by incubation with low agitation at $37^{\circ} \mathrm{C}$. Dilution ranged from 20,000 infected RBCs (iRBCs) $/ \mu \mathrm{l}$ to $0.1 \mathrm{iRBCs} / \mu \mathrm{l}$. For microscopy examination, thin blood films were made from each dilution after careful suspension, while thick blood films were made for the lower parasitaemia. Three slides were prepared for each dilution and read by two microscopists in a blind manner. Parasitaemia was determined by counting fields with $100 \mathrm{RBCs}$, and the total level was expressed in $\mu \mathrm{L}$ of blood corresponding of $5.106 \mathrm{RBCs} / \mu \mathrm{L}$. A linear regression curve based on mean parasitaemia levels was established in order to determine the parasitaemia, and a standard curve was generated using recombinant pLDH to assess the correlation between parasitaemia and pLDH concentration.

\section{Recombinant pLDH}

A synthetic gene (Genscript, NJ, USA) encoding for $P$. falciparum $\mathrm{pLDH}$ protein was inserted in the expression vector pMAL C2X (New England Biolabs, Ipswich, MA, USA) containing the maltose binding protein (MBP) fusion partner sequence. The protein was expressed in Escherichia coli and purified by affinity chromatography on amylose resin. The protein was used to generate the standard curve.

\section{Antibodies detection}

Antibody screening was performed using a homemade ELISA-antibody test. The serological ELISA test was derived from a commercial assay exhibiting high specificity and analytical sensitivity, namely 96.7 and $93.1 \%$, respectively [27]. The test was based on binding of antiPlasmodium antibodies present in serum or plasma samples to antigens immobilized on 96-well plates, with the antigen being an extract of in vitro $P$. falciparum culture (3D7 strain). Due to antigenic community, antibodies to other Plasmodium species may be detected. The diluent solution containing PBS and Tween $0.1 \%$,
$(125 \mu \mathrm{L})$ was poured into each well, followed by $25 \mu \mathrm{L}$ of test plasma. On the same plate, $25 \mu \mathrm{L}$ positive control and negative control were poured in single and triplicate wells, respectively. The plate incubated for $60 \mathrm{~min}$ at $37^{\circ} \mathrm{C}$ before being washed five times. One hundred $\mu \mathrm{L}$ of horseradish peroxidase-conjugated rabbit anti-human IgG polyclonal antibodies (Sigma-Aldrich, St Quentin, France) were added to each well, and the plate incubated for a further $30 \mathrm{~min}$ at $37^{\circ} \mathrm{C}$. The wells were washed five times, and 100 $\mu \mathrm{L}$ of TMB plus substrate solution (tetramethylbenzidine) (Kem-en-tec, Denmark) were added to each well. The plate was covered and incubated in the dark for $15 \mathrm{~min}$ at $37^{\circ} \mathrm{C}$. Finally, $50 \mu \mathrm{L}$ of $0.5 \mathrm{M}$ sulphuric acid was added to each well, and absorbance was read within $15 \mathrm{~min}$ at 450 $\mathrm{nm}$, with a reference wave-length of $620 \mathrm{~nm}$. Test validation required that the positive OD be $>0.500$ and the negative $\mathrm{OD} \geq 0.200$. The cut-off value was calculated by multiplying the negative control wells' average OD by four. The antibody $(\mathrm{Ab})$ index of each sample was calculated by dividing its OD value by the cut-off value. The sample was considered positive if the $\mathrm{Ab}$ index was $>1.0$, equivocal if the $\mathrm{Ab}$ index was between 0.8 and 1.0, and negative if the $\mathrm{Ab}$ index was $\leq 0.8$. The test was not able to distinguish between antibodies directed to either $P$. falciparum, $P$. vivax, $P$. malariae, or P. ovale.

\section{Data analysis}

The data was analysed by the chi-squared, Bartlett's chisquared, and Kruskal-Wallis tests using Sigma Plot 2001 software (Sigma Plot 2001 Statistical Analysis Software, San Jose, CA, USA) and Epi Info version 3.5.1. The results were expressed as mean \pm standard deviation. Statistical significance was reached if $\mathrm{P}<0.05$, with a $95 \%$ confidence interval $(\mathrm{CI})$.

\section{Results \\ pLDH ELISA performance \\ Sensitivity}

Among the 266 malaria patients, the antigen detection test was able to detect 250 cases while missing 16, resulting in a sensitivity of $94 \%$, with borderline results included as positive in the calculations. Nine cases of $P$. falciparum were missed, five of them were treated for malaria before the blood examination and the four remaining patients did not provide any information's regarding a previous treatment. Seven cases of $P$. ovale were also missed may be due to the fact that the monoclonal antibodies for $\mathrm{pLDH}$ fail to detect some $P$. ovale strains. However this point was not explored in our study and not discussed. The antibody detection test was able to detect only 220 cases (Table 1 ). Moreover, the combined tests detected 261 positive cases and missed only five, namely a $P$. falciparum-infected patient with prior antimalarial treatment, and four $P$. ovale-infected patients 


\begin{tabular}{|c|c|c|c|}
\hline Species & Microscopy & pLDH ELISA & Antibody ELISA \\
\hline P. falciparum & 239/266 (89.9\%) & $230 / 239$ & 199/239 \\
\hline P. ovale & 19/266 (7.1\%) & $12 / 19$ & $14 / 19$ \\
\hline P. malariae & $6 / 266$ (2.25\%) & $6 / 6$ & $5 / 6$ \\
\hline Mixed infection ${ }^{1}$ & 2/266 (0.75\%) & $2 / 2$ & $2 / 2$ \\
\hline Total & & $250 / 266$ (94\%) & $220 / 266$ (82.7\%) \\
\hline
\end{tabular}

${ }^{1}$ Mixed infections are $P$. ovale with $P$. falciparum.

(Table 2). Specificity for malaria-risk blood donors and not-exposed-to-malaria blood donors was 99.6 and $99.5 \%$, respectively, with an overall specificity of $99.57 \%$. The pLDH assay's positive and negative predictive values were $94.3 \%$ and $99.5 \%$, respectively.

\section{Detectability}

The parasite detection threshold for microscopy and pLDH Elisa test was calculated using a range of infected iRBCs and recombinant pLDH. The calculated parasitaemia in diluted iRBCs ranged from 24,676 iRBCs/ $\mu$ to $0.0005, \mathrm{iRBCs} / \mu \mathrm{l}$ (extrapolated value), with a linear regression line and 0.977 correlation coefficient. The detection limit was 1 parasite $/ \mu \mathrm{L}$ for the $\mathrm{pLDH}$ ELISA, which corresponds to $0.08 \mathrm{ng} / \mathrm{ml}$ of recombinant $\mathrm{pLDH}$. The detection threshold with the standard curve using the recombinant $\mathrm{pLDH}$ was $0.125 \mathrm{ng} / \mathrm{mL}$, the curve being linear up to $2.5 \mathrm{ng} / \mathrm{mL}$ (Figure 1) (Table 3).

\section{pLDH assay evaluation in a population of Benin blood donors \\ Characteristics of the blood donor population}

Blood samples from 2,515 donors were collected over a ten-month period covering four seasons: LRS, SDS, SRS, and LDS. The samples were distributed as follows: 590/ 2,515 (23.5\%) in LRS, 409/2,515 (16.3\%) in SDS, 630/ $2,515(25.0 \%)$ in SRS and 886/2,515 (35.2\%) (Table 4). The donors enrolled in this study were young, with a median age of $31 \pm 9$ years. Their age distribution showed that young people between 25 and 40 years were the most represented, with 1,379/2,515 (54.8\%). Sex

Table 2 Comparison of pLDH ELISA (Ag) and antibody ELISA (Ab) detection in a population of microscopically confirmed malaria patients $(n=266)$

\begin{tabular}{lccc}
\hline & Ab Pos & Ab Neg & Total \\
\hline Ag pos & 209 & 41 & $\mathbf{2 5 0}$ \\
Ag neg & 11 & $5^{*}$ & $\mathbf{1 6}$ \\
TOTAL & $\mathbf{2 2 0}$ & $\mathbf{4 6}$ & $\mathbf{2 6 6}$ \\
\hline
\end{tabular}

*One case of $P$. falciparum infection treated prior to the parasitological diagnosis and four $P$. ovale cases. distribution exhibited a strong male predominance with 2,027/2,515 (80.60\%) men versus 488/2,515 (19.40\%, 95\% CI: 17.9-21.0\%) women. Among the donors, 1,989/2,515 slept under a mosquito net (79.1\%, 95\% CI: 77.4-80.6\%). The majority of donors, namely 1,521/2,515 (60.47\%, 95\% CI: 57.4-61.2\%), reported having had episodes of malaria, and the predominant clinical prior malaria expression was a single suspected access in 1,387/2,515 (55.1\%, 95\% CI: 53.2-57.1\%), whereas 51 donors (2.0\%, 95\% CI: $1.5-2.7 \%)$ reported a severe attack requiring hospitalization in the last year. In total, 83 donors $(3.3 \%$, 95\% CI: 2.7-4.1) had both single and severe malaria attacks, whereas 994 donors (39.52\%) reported having no malaria attack during the preceding year. Among the 1,521 donors with suspected malaria episodes, antimalarial treatment was administered to 509 (33.46\%) in health facilities during the last malaria attack, whereas $1,012 / 1,521(66.53 \%)$ were treated as outpatients. Most blood donors did not receive chemoprophylaxis but performed a sort of prevention when they experienced "signs of malaria". Self-prevention against malaria was undertaken by $1,537 / 2,515$ donors (61.11\%) who took anti-malarial drugs or herbal tea, whereas 978/2,515 $(38.88 \%)$ did not undertake any prevention. The drugs used were quinine 567/2,515 (22.5\%, 95\% CI: 20.9-24.2\%), artemisinin-based combination 510/2,515 (20.3\%, 95\% CI: 18.7-21.9\%), herbal teas 336/2,515 (13.4\%, 95\% CI: $12.1-$ 14.8\%), and Fansidar ${ }^{\circledR} 124 / 2,515$ (4.9\%, 95\% CI: 4.1-5.9\%).

\section{Microscopic prevalence of Plasmodium}

Among the 2,515 blood donors, the rate of asymptomatic Plasmodium carriage measured by microscopy was 295/2,515 (11.72\%, 95\% CI: 10.5-13.1\%). Three Plasmodium species were detected: $P$. falciparum was identified in all infection cases, $P$. malariae in 14 mixed infection cases $(5.1 \%)$, and $P$. ovale in one mixed infection case (0.34\%) (Table 4). The study findings revealed a higher percentage of infection among males 252/295 (85.42\%) compared to females 43/295 (14.6\%). Microscopic prevalence varied according to seasons: $P$. falciparum and $P$. malariae detection was constant during all seasons (Table 4), whereas P. falciparum density was very low and highly variable. Among the 295 positive donors, 238 (80.67\%) exhibited a parasite density between seven and 100 parasites per microliter $(\mathrm{p} / \mu \mathrm{L})$, with $0.3 \%$ exhibiting a density $>5,000 \mathrm{p} / \mu \mathrm{L}$ (Table 5 ).

\section{Malaria antibodies prevalence}

Plasmodium antibody detection by ELISA in 2,515 donors showed a high positivity rate with a prevalence of 73.9\% (1,859/2,515) (95\% CI: 72.1-75.6\%). Equivocal results were found in $13.1 \%$ of samples $(329 / 2,515)$, and there were no detectable antibodies in 327 donors (13.0\%, 95\% CI: 11.7-14.4\%) (Table 6B). Antibody prevalence 

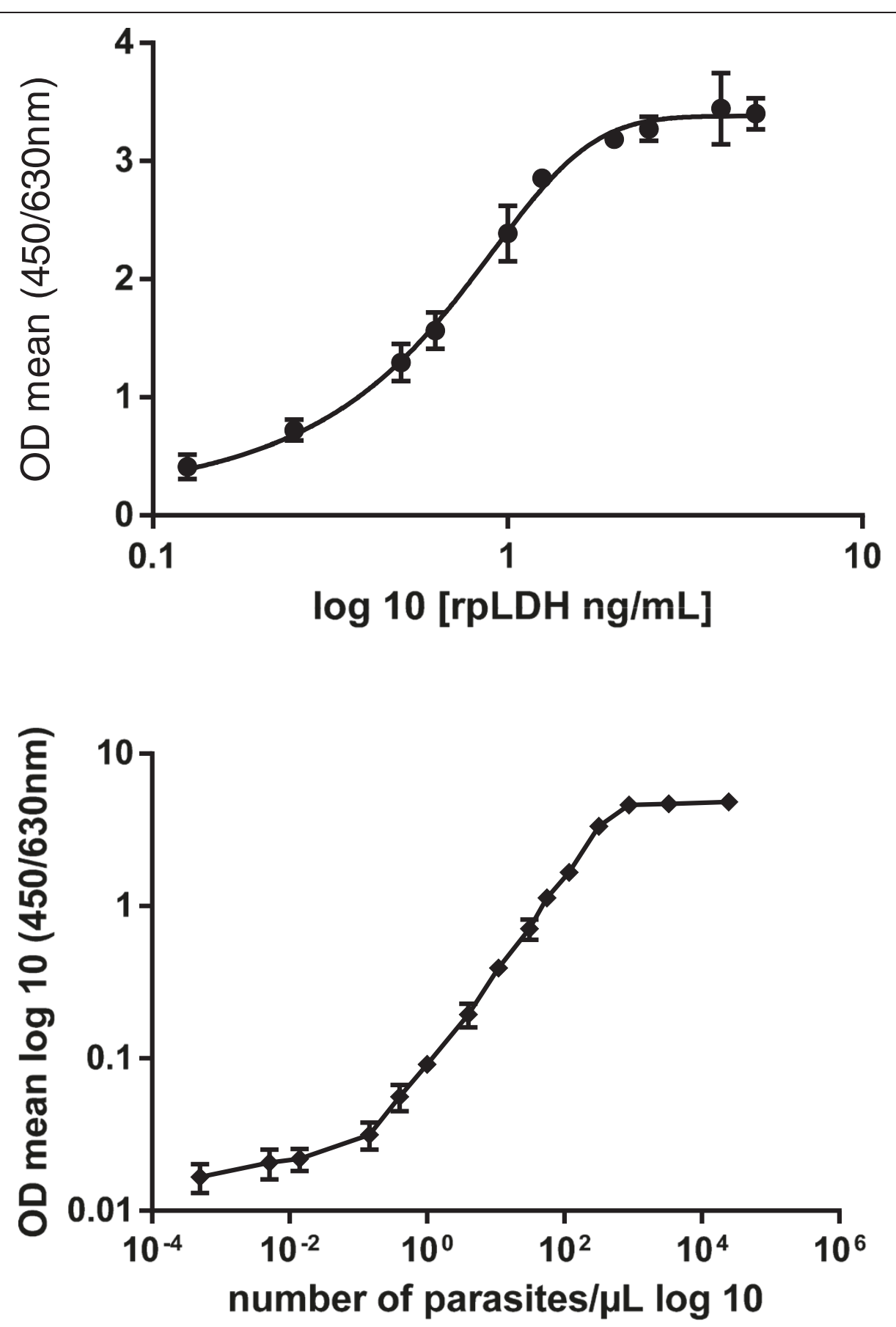

Figure 1 pLDH detectability for Plasmodium falciparum and recombinant pLDH. Standard curve for a range of ten assays for rpLDH and 15 assays for Pf iRBCs. Detectability was $2.5 \mathrm{ng} / \mathrm{mL}$ for rpLDH and one parasite per $\mu \mathrm{L}$ for RBC.

differed significantly according to the season $(\mathrm{P}<0.05)$ (Table 7 and Figure 2).

pLDH prevalence in Benin blood donors

pLDH antigen was found in $38.4 \%$ of blood donors (966/2,515) (95\% CI: 36.5-40.3\%), with equivocal results $(n=622 / 2,515,24.7 \%)$ considered as positive for the calculation. The prevalence of pLDH antigen confirmed by microscopy was $230 / 966(23.8 \%)$. The only sample that was positive by microscopy but negative by pLDH detection was a $P$. malariae infection case, notably a malaria-antibody positive sample (Table 6A).

pLDH antigen prevalence varied significantly according to the season $(\mathrm{P}<0.05)$ (Table 7 and Figure 2$)$. 
Table 3 Detectability of pLDH evaluated with a recombinant pLDH (A) and Plasmodium falciparum-infected red blood cells (Pf iRBCs) (B)

\begin{tabular}{|c|c|c|}
\hline \multicolumn{3}{|l|}{$\bar{A}$} \\
\hline $\mathrm{rpLDH}$ ng/mL & Index & SD \\
\hline 0 & 0.335 & 0.071 \\
\hline 0.125 & 1.465 & 0.365 \\
\hline 0.25 & 2.650 & 0.312 \\
\hline 0.5 & 4.595 & 0.553 \\
\hline 0.625 & 5.550 & 0.550 \\
\hline 1 & 8.464 & 0.830 \\
\hline 1.25 & 10.129 & 0.149 \\
\hline 2 & 11.299 & 0.145 \\
\hline 2.5 & 11.607 & 0.358 \\
\hline 4 & 12.11 & 1.067 \\
\hline 5 & 12.057 & 0.461 \\
\hline \multicolumn{3}{|l|}{ B } \\
\hline PARASITE/ $\mu \mathrm{L}$ & Index & SD \\
\hline 0 & 0.194 & 0.061 \\
\hline 0.0005 & 0.165 & 0.036 \\
\hline 0.0051 & 0.205 & 0.045 \\
\hline 0.0141 & 0.217 & 0.036 \\
\hline 0.1455 & 0.312 & 0.064 \\
\hline 0.4 & 0.556 & 0.110 \\
\hline 1 & 0.908 & 0.100 \\
\hline 4 & 1.922 & 0.335 \\
\hline 11 & 3.886 & 0.310 \\
\hline 31 & 7.017 & 1.073 \\
\hline 56 & 11.227 & 1.000 \\
\hline 117 & 16.523 & 1.609 \\
\hline 319 & 33.032 & 3.073 \\
\hline 872 & 45.664 & 2.435 \\
\hline 3,298 & 46.396 & 2.353 \\
\hline 24,676 & 47.630 & 2.444 \\
\hline
\end{tabular}

Table 5 Variation of parasitaemia in the $\mathbf{2 9 5}$ positive donors by microscopy

\begin{tabular}{ccc}
\hline P/ $\boldsymbol{\mu L}$ & Frequency & Percent (\%) \\
\hline $7-100$ & 238 & 80.7 \\
$101-500$ & 1 & 15.9 \\
$501-5,000$ & 9 & 3.1 \\
$>5,000$ & 1 & 0.3 \\
Total & $\mathbf{2 9 5}$ & \\
\hline
\end{tabular}

Interestingly enough, $25.1 \%$ of the blood donors with equivocal pLDH index were microscopically negative. However, a similar percentage was observed in microscopically positive blood donors, suggesting that the grey zone was correctly set up for pLDH (Table 8). The absence of antibodies in the blood of patients with detectable parasitaemia was noted in 30 cases $(30 / 295 ; 10.2 \%)$, but 20 of them tested positive when using the pLDH ELISA. In 744/2,515 (29.6\%) cases, both antibody and antigen were present in samples, and 199 samples were positive $(n=112)$ or equivocal $(n=87)$ for $\mathrm{pLDH}$ and negative for antibodies (Table 9).

\section{Discussion}

In this study, a commercialized pan Plasmodium LDH ELISA was shown to exhibit optimal performances as to both PPV and NPV. The assay was able to detect one parasite per $\mu \mathrm{L}$, which is the lowest detectability reported in the scientific literature to date. This detection threshold is much lower than that of the already commercialized rapid test [28], which is estimated at 50 parasites $/ \mu \mathrm{L}$. An ELISA-based assay would theoretically lower that detectability. Indeed, another ELISA assay based on HRP2 detection was able to detect 11.7 parasites/ $\mu \mathrm{L}$, which is equivalent to $6 \mathrm{ng} / \mathrm{ml}$ of $\mathrm{rPfHRP} 2$ [29]. The authors of this study conducted in Kenya reported that the microscopic detection limit was three parasites/ $\mu \mathrm{L}$, with a lower reproducibility. The ELISA assay, which is able to detect in vitro lower amounts of $P$. falciparum and other species, would thus be an interesting tool to detect the presence of Plasmodium in a given blood

Table 4 Distribution of microscopically detected Plasmodium species in a population of 2,515 blood donors during the four seasons: long rainy season (LRS), short dry season (SDS), short rainy season (SRS), and long dry season (LDS)

\begin{tabular}{|c|c|c|c|}
\hline Sampling period & Positive/donors & $\begin{array}{l}\text { Positivity } \\
\text { rate }\end{array}$ & Prevalence species \\
\hline & $(\mathrm{N}=\mathbf{2 5 1 5})$ & (\%) & $(\mathrm{N}=295)$ \\
\hline LRS (May-June-July) & $67 / 590$ & 11.4 & P. falciparum (64/67) 95.52\%; *P. malariae (3/67) 4.47\%; P. ovale (0) $0.0 \%$ \\
\hline SDS (August -September) & $48 / 409$ & 11.7 & P. falciparum (45/48) 93.75\%; *P. malariae (3/48) 6.25\%; P. ovale (0) $0.0 \%$ \\
\hline SRS (October-November) & $100 / 630$ & 16.0 & P. falciparum (96/100) 96.0\%; ${ }^{*}$ P. malariae $(3 / 100) 3.0 \% ;{ }^{*}$ P. ovale $(1 / 100) 1.0 \%$ \\
\hline LDS (December-January-February) & $80 / 886$ & 9.0 & P. falciparum (75/79 (93.75\%; *P. malariae (5/80) 6.25\%; P. ovale (0) $0.00 \%$ \\
\hline TOTAL & $295 / 2,515$ & 11.72 & $\begin{array}{c}\text { P. falciparum (280/295) 95.0\%; }{ }^{*} \text { P. malariae (14/295) 5.0\%; }{ }^{*} \text { P. ovale }(1 / 295) 0.34 \% \\
\text { Mixed infection (15/295) 5.10\% }\end{array}$ \\
\hline
\end{tabular}

*Mixed infection. 
Table 6 Comparison of microscopy with pLDH ELISA (A) and antibody ELISA (B) in blood donors

\begin{tabular}{cccccc}
\hline \multirow{2}{*}{ A } & \multicolumn{4}{c}{ pLDH ELISA } \\
\cline { 3 - 6 } & & Positive & Equivocal & Negative & TOTAL \\
\hline \multirow{3}{*}{ Microscopy } & Positive & 230 & 64 & 1 & 295 \\
& Negative & 736 & 558 & 926 & 2,220 \\
& TOTAL & 966 & 622 & 927 & 2,515 \\
\hline \multirow{2}{*}{ B } & & \multicolumn{4}{c}{ Antibody ELISA } \\
\cline { 3 - 6 } & & Positive & Equivocal & Negative & TOTAL \\
\hline \multirow{3}{*}{ Microscopy } & Positive & 231 & 34 & 30 & 295 \\
& Negative & 1,628 & 295 & 297 & 2,220 \\
& TOTAL & 1,859 & 329 & 327 & 2,515 \\
\hline
\end{tabular}

donor population. However the reagent failed to detect seven cases of $P$. ovale out of 19 cases. This defect could due to the incapability of the assay to detect the pLDH from some strains of $P$. ovale [30]. The use of a commercialized pan Plasmodium LDH ELISA was evaluated in a large blood donor population from southern Benin. The test results were compared to those obtained using microscopy and to those pertaining to malaria-antibody prevalence. The study aimed to determine whether $\mathrm{pLDH}$ detection was sensitive enough to identify low parasitaemia levels, which are frequently the case in asymptomatic malaria blood donors, and to assess the prevalence of positive pLDH blood donations in southern Benin.

This study enrolled 2,515 blood donors from six southern Benin departments, with similar malaria epidemiological features. These departments exhibit a wet tropical climate, with two rainy seasons and two dry seasons. In these regions, malaria transmission is permanent, with an increase during the rainy season [16]. The most transmitted species are P. falciparum (97.10\%) and P. malariae $(2.00 \%)[15,31]$. The figures reported in the literature are similar to those found in this study, with a 95.0\% prevalence of $P$. falciparum and $4.7 \%$ prevalence of $P$. malariae. The overall prevalence of asymptomatic Plasmodium carriage here was $11.72 \%$. Plasmodium falciparum parasite density proved to be very low and highly variable. Two-third of these positive donors had a parasite density between seven and 100 parasites per $\mu \mathrm{L}$. Reported prevalence rates in Benin appear to be similar to those reported by some authors in neighbouring Nigeria, $10.2 \%$ [32], or in Ghana, 10.20\%. [33]. In this study, $P$. falciparum was identified in all infected cases, $5.0 \%$ of which were mixed $P$. malariae infections. These figures are in line with the reported presence of $P$. falciparum in all infected cases and the $2.3 \%$ rate of mixed infection in Nigeria [3]. However, these results differ from those of other authors who reported higher parasite prevalence rates, namely $50.7 \%$ in Burkina Faso [34], $55 \%$ in Nigeria [13,23], and $33.52 \%$ in Benin [4].

The study findings highlighted a higher infection rate in male blood donors compared to female ones (85.42 vs $14.6 \%)$. In a study conducted in Colombia [35], males were also found to be more infected with Plasmodium ssp. than females, 59 and 41\%, respectively. Nevertheless, in a 1994 publication by Vlassoff et al. [36], Ghanaian women were found to have higher infection rates than Ghanaian men.

According to the study, the risk of Plasmodium transmission through blood transfusion is accounted for by the persistence of malaria parasites in blood. Canonical knowledge indicates that $P$. falciparum may persist in blood for one year, $P$. ovale for three years, and $P$. malariae for even longer time periods before causing malaria [20,32]. Similarly, the fact that malarial parasites may survive in $\mathrm{RBCs}$ at refrigerator temperatures $\left(2-6^{\circ} \mathrm{C}\right)$ for days or weeks leads to the original exclusion of all blood donors who could represent a potential risk in TTM [6,7]. This observation supports some authors' suggestions, namely to implement Plasmodium screening prior to blood donation in Africa $[3,37]$.

In non-malaria-endemic area, the malaria antibody detection test will result in rejecting blood donation in case of positivity. However, in malaria-endemic countries, such as Benin, this tool is useless. Indeed, malaria antibody prevalence was $87 \%$ ( $74.0 \%$ positive and $13 \%$ equivocal) in the study's blood donor populations. This prevalence was similar to that reported for Senegal, with $65.33 \%$ being positive and $6.86 \%$ equivocal [19]. Therefore, this high antibody seroprevalence in blood donors invalidates the marker's use for detecting Plasmodium-infected blood donations. It is interesting to note that detectable Plasmodium parasites were found in blood donors but without antibodies, suggesting an acute infection.

In this study, $38.4 \%$ of the blood donors were tested positive for $\mathrm{pLDH}$ antigen, with a $4.2 \%$ rate of equivocal results. The pLDH antigen detection results differed from those found by Diop et al. in Senegal, who reported a detection rate of $0.53 \%$ for $\mathrm{pLDH}$, with a $2.23 \%$ rate of

Table 7 Positivity rate of pLDH ELISA and antibody ELISA tests according to infecting donors by Plasmodium during the seasons, long rainy season (LRS), short dry season (SDS), short rainy season (SRS), and long dry season (LDS)

\begin{tabular}{ccccccc}
\hline & & \multicolumn{4}{c}{ Season } \\
\cline { 3 - 6 } & & LRS & SDS & SRS & LDS & TOTAL \\
\hline pLDH ELISA & POS & $142(24.1 \%)$ & $206(50.3 \%)$ & $323(51.3 \%)$ & $295(33.3 \%)$ & $966(38.4 \%)$ \\
Antibody ELISA & POS & $453(76.7 \%)$ & $288(70.1 \%)$ & $368(58.4 \%)$ & $751(84.7 \%)$ & $1.859(73.9 \%)$ \\
\hline
\end{tabular}




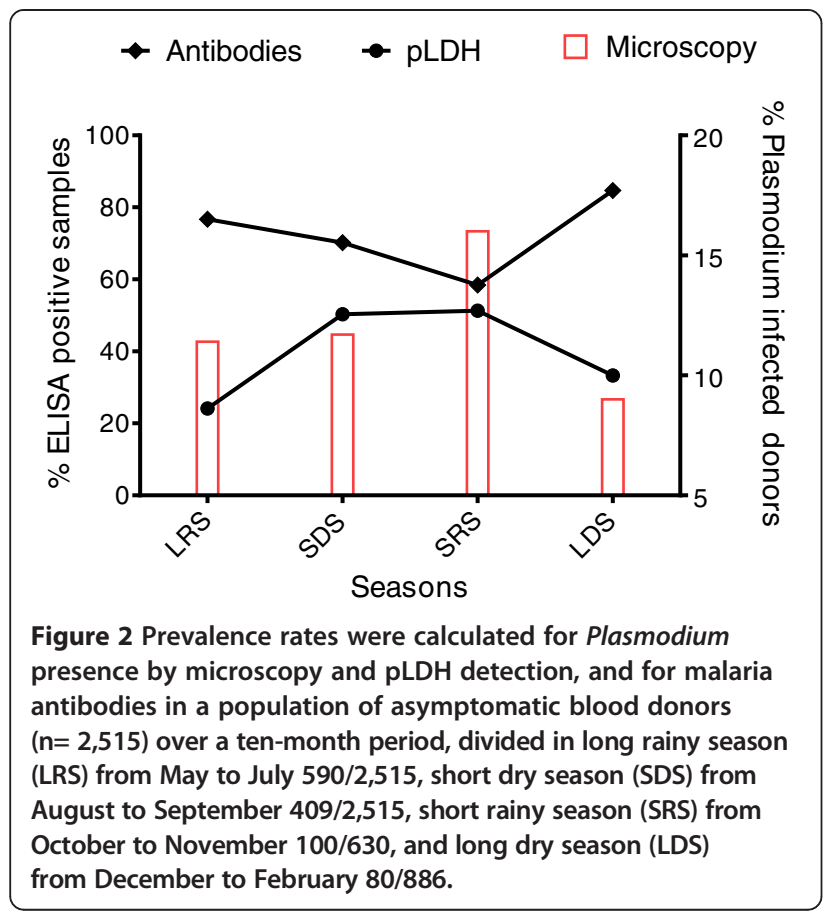

doubtful cases [19]. However, the malaria epidemiology in Senegal is highly different from that in Benin. The low parasitaemia level may escape microscopic examination, while being dependent on the reader's skill. The pLDH ELISA exhibited a detectability threshold lower than that of the other methods, with the exception of PCR. This finding suggests that pLDH detection could be a valid tool for blood bank settings in order to identify blood donation with a detectable parasitic load of at least one parasite per $\mu \mathrm{L}$.

The questionnaire's effectiveness helped to identify certain risk factors associated with pLDH antigen carriage, such as blood donations during high transmission periods. Based on the study results it was noticed that seasons influenced both microscopic and pLDH prevalence. Others factors likely impact these markers, such as the malaria prevention method used by blood donors, i.e. drugs, herbal teas, etc. The limits of selecting blood

Table 8 Distribution of pLDH ELISA in microscopically positive and negative blood donors

\begin{tabular}{cccccc}
\hline $\begin{array}{c}\text { pLDH ELISA } \\
\text { index }\end{array}$ & \multicolumn{2}{c}{ Positive donors } & & \multicolumn{2}{c}{ Negative donors } \\
\cline { 2 - 3 } \cline { 5 - 6 } \cline { 5 - 6 } & Frequency & Percent (\%) & & Frequency & Percent (\%) \\
\hline 10-20 & 45 & 15.3 & & 2 & 0.01 \\
$1-10$ & 24 & 8.1 & & 2 & 0.01 \\
0.7-0.99 & 161 & 54.6 & & 737 & 33.2 \\
Negative & 1 & 21.7 & & 558 & 25.1 \\
TOTAL & $\mathbf{2 9 5}$ & 0.3 & & 921 & 41.5 \\
\hline
\end{tabular}

Table 9 Antibody ELISA and pLDH ELISA comparative results for Benin blood donors

\begin{tabular}{lccccc}
\hline & & \multicolumn{4}{c}{ pLDH ELISA } \\
\cline { 3 - 6 } & & Positive & Equivocal & Negative & Total \\
\hline Antibody ELISA & Positive & $\mathbf{7 4 4}$ & 442 & 673 & 1,859 \\
& Equivocal & 110 & 94 & 126 & 329 \\
& Negative & 112 & 87 & 128 & 327 \\
& TOTAL & 966 & 622 & 927 & 2,515 \\
\hline
\end{tabular}

donors through medical questionnaires were highlighted by the study findings, as $38.4 \%$ positive donors for ELISA pLDH had met the selection criteria and gave their blood. It is, therefore, logical to propose implementation of Plasmodium screening in blood donation, which was not performed in Benin due to costs and lack of technique reliability, still keeping in mind that blood donors who report an undiagnosed and untreated febrile illness consistent with malaria should be deferred until asymptomatic and off the treatment. In a recent cost effectiveness analysis study, Rajab et al. [38] concluded that pretransfusion screening would be less costly than the recommended artemisinin-based combination used for prophylaxis in blood recipients, especially children and pregnant women.

\section{Conclusion}

Malaria and transfusion is a "neglected subject coming back to the fore front", as recently discussed by Allain [39]. The microscopy and antibody detection techniques are not suited for mass screening in a blood transfusion centre in endemic areas. Based on this study results, the pLDH antigen detection ELISA for Plasmodium could be an interesting tool for blood donation qualification in order to ensure blood safety in malaria-endemic areas. One of the test's advantages is that false-positives are exceptional, unlike the HRP2 (histidine-rich-protein)-based tests, which can remain positive for two weeks after parasite clearance. HRP2 may also be absent in malaria infection cases. pLDH antigen ELISA detection in the blood is efficient when there is a parasite [40]. Some pitfalls were observed in the study. In some $P$. ovale infection cases the assay failed to detect them. Similarly, if the donors had taken self-treatment measures prior to blood donation, malaria infection was masked and pLDH detection failed. In conclusion, routine screening of all donated blood would prevent infected blood donation and increase the donation's safety for fragile recipients, such as children and pregnant women. It would also be instrumental in reducing unnecessary medical treatments in recipients and contribute to lower Plasmodium resistance. The main problem remains the feasibility of rejecting positive donations in term of blood availability in endemic area where blood transfusion needs are presently increasing. 


\section{Competing interests}

The authors declare that they have no competing interests.

\section{Authors' contributions}

PSA, CDL, AS and EC designed the study, and performed the acquisition, the analysis and the interpretation of data. NWC, SP, TA, CDA, LA and AB were involved in the acquisition, the analysis and interpretation of data. All authors have given a final approval to the manuscript.

\section{Acknowledgements}

This work was supported by the Project CONNECTUS on Transfusion malaria diagnosis. S.P Atchade was supported by a grant of the Benin Government.

\section{Author details}

${ }^{1}$ Institut de Parasitologie et de Pathologie Tropicale (IPPTS) - Fédération de Médecine Translationnelle, Université de Strasbourg, Strasbourg, France. 'Laboratoire de Biochimie et de Biologie Moléculaire, Université d'Abomey Calavi, 04 BP 0320 Cotonou, Bénin. ${ }^{3}$ Public Health Wales Microbiology, Public Health Wales NHS Trust, Cardiff, UK. ${ }^{4}$ Agence Nationale pour la Transfusion Sanguine (Ministère de la Santé), 01 B.P. 511 Cotonou, Bénin.

Received: 21 March 2013 Accepted: 6 July 2013

Published: 8 August 2013

\section{References}

1. Woolsey: Transfusion for pernicious anaemia: two cases. Ann Surg 1991, 53:132-135.

2. Wylie BR: Transfusion transmitted infection: viral and exotic diseases. Anaesth Intensive Care 1993, 21:24-30.

3. Uneke CJ, Ogbu O, Nwojiji V: Potential risk of induced malaria by blood transfusion in South-eastern Nigeria. McGill J Med 2006, 9:8-13.

4. Kinde G, Oke J, Gnahoui I, Massougbodji A: [The risk of malaria transmission by blood transfusion at Cotonou, Benin](in French). Sante 2000, 10:389-392.

5. Chattopadhyay R, Majam VF, Kumar S: Survival of Plasmodium falciparum in human blood during refrigeration. Transfusion 2011, 51:630-635.

6. Sazama K: Prevention of transfusion-transmitted malaria: is it time to revisit the standards? Transfusion 1991, 31:786-788.

7. Candolfi E: [Transfusion-transmitted malaria, preventive measures](in French). Transfus Clin Biol 2005, 12:107-113.

8. Garraud O, Assal A, Pelletier B, Danic B, Kerleguer A, David B, Joussemet M, de Micco P: Overview of revised measures to prevent malaria transmission by blood transfusion in France. Vox Sang 2008, 95:226-231.

9. Kirchgatter K, Del Portillo HA: Clinical and molecular aspects of severe malaria. An Acad Bras Cienc 2005, 77:455-475.

10. Reesink HW, Panzer S, Wendel S, Levi JE, Ullum H, Ekblom-Kullberg $S$, Seifried E, Schmidt M, Shinar E, Prati D, Berzuini A, Ghosh S, Flesland O, Jeansson S, Zhiburt E, Piron M, Sauleda S, Ekermo B, Eglin R, Kitchen A, Dodd RY, Leiby DA, Katz LM, Kleinman S: The use of malaria antibody tests in the prevention of transfusion-transmitted malaria. Vox Sang 2011, 98:468-478

11. Kitchen A, Mijovic A, Hewitt P: Transfusion-transmitted malaria: current donor selection guidelines are not sufficient. Vox Sang 2005, 88:200-201.

12. Reesink HW: European strategies against the parasite transfusion risk. Transfus Clin Biol 2005, 12:1-4.

13. Epidi TT, Nwani CD, Ugorji NP: Prevalence of malaria in blood donors in Abakaliki Metropolis, Nigeria. Sci Res Essays 2008, 3:162-164.

14. Schindler HC, Montenegro L, Montenegro R, Carvalho AB, Abath FG, Jaureguiberry G: Development and optimization of polymerase chain reaction-based malaria diagnostic methods and their comparison with quantitative buffy coat assay. Am J Trop Med Hyg 2001, 65:355-361.

15. Akogbeto M: [Entomological study on the malaria transmission in coastal and lagoon areas: the case of a village built on a brackish lake](in French). Ann Soc Belg Med Trop 1995, 75:219-227.

16. Yadouleton AW, Asidi A, Djouaka RF, Braima J, Agossou CD, Akogbeto MC: Development of vegetable farming: a cause of the emergence of insecticide resistance in populations of Anopheles gambiae in urban areas of Benin. Malar J 2009, 8:103.

17. Ministère de la Santé (Ed): Annuaire des statistiques sanitaires année 2006; 2006 18. WHO: World Malaria Report 2012. Geneva: World Health Organization; 2012
19. Diop S, Ndiaye M, Seck M, Chevalier B, Jambou R, Sarr A, Dieye TN, Toure $A O$, Thiam D, Diakhate L: [Prevention of transfusion transmitted malaria in endemic area] (in French). Transfus Clin Biol 2009, 16:454-459.

20. Kitchen AD, Chiodini PL: Malaria and blood transfusion. Vox Sang 2006 90:77-84.

21. Carme B, Kenmogne D, Copin N, Mbitsi A: [Plasmodium prevalence and parasitic burden in blood donors of Brazzaville, Congo](in French). Ann Soc Belg Med Trop 1993, 73(3):179-187.

22. Achidi EA, Perlmann $H$, Berzins K: Asymptomatic malaria parasitaemia and seroreactivities to Plasmodium falciparum antigens in blood donors from Ibadan, south-western Nigeria. Ann Trop Med Parasitol 1995, 89:601-610.

23. Okocha EC, Ibeh CC, Ele PU, Ibeh NC: The prevalence of malaria parasitaemia in blood donors in a Nigerian teaching hospital. $J$ Vector Borne Dis 2005, 42:21-24.

24. Harani MS, Beg MA, Khaleeq L, Adil SN, Kakepoto GN, Khurshid M: Role of ICT malaria immunochromatographic test for rapid diagnosis of malaria. J Pak Med Assoc 2006, 56:167-171.

25. Oduola AM, Omitowoju GO, Sowunmi A, Makler MT, Falade CO, Kyle DE, Fehintola FA, Ogundahunsi OA, Piper RC, Schuster BG, Milhous WK: Plasmodium falciparum: evaluation of lactate dehydrogenase in monitoring therapeutic responses to standard antimalarial drugs in Nigeria. Exp Parasitol 1997, 87:283-289.

26. Trager $W$, Jensen JB: Human malaria parasites in continuous culture. Science 1976, 193:673-675.

27. Doderer C, Heschung A, Guntz P, Cazenave JP, Hansmann Y, Senegas A, Pfaff AW, Abelrahman T, Candolfi E: A new ELISA kit which uses a combination of $P$. falciparum extract and recombinant $P$. vivax antigens as an alternative to IFAT for detection of malaria antibodies. Malar $J$ 2007, 6:19.

28. Wilson ML: Malaria rapid diagnostic tests. Clin Infect Dis 2012, 54:1637-1641.

29. Kifude CM, Rajasekariah HG, Sullivan DJ Jr, Stewart VA, Angov E, Martin SK, Diggs CL, Waitumbi JN: Enzyme-linked immunosorbent assay for detection of Plasmodium falciparum histidine-rich protein 2 in blood, plasma, and serum. Clin Vaccine Immunol 2008, 15:1012-1018.

30. Calderaro A, Piccolo G, Perandin F, Gorrini C, Peruzzi S, Zuelli C, Ricci L, Manca N, Dettori G, Chezzi C, Snounou G: Genetic polymorphisms influence Plasmodium ovale PCR detection accuracy. J Clin Microbiol 2007, 45:1624-1627.

31. Carnevale P, Mouchet J: [Vector control and malaria control](in French). Med Trop (Mars) 1990, 50:391-398.

32. Erhabor O, Ok O, Awah I, Uko KE, Charles AT: The prevalence of Plasmodia parasitaemia among donors in the Niger delta of Nigeria. Trop Doct 2007, 37:32-34.

33. Owusu-Ofori AK, Parry C, Bates I: Transfusion-transmitted malaria in countries where malaria is endemic: a review of the literature from subSaharan Africa. Clin Infect Dis 2010, 51:1192-1198.

34. Guiguemde T, Sanou M, Ouedraogo J, Coulibaly N, Ghary A, Coulibaly S: Le paludisme et la transfusion: une étude portant sur les donneurs de la banque de sang de l'hôpital de Bobo-Dioulasso (burkina Fasso). Malaria Infect Dis Afr 1995, 2:9.

35. Bonilla $E$, Rodriguez A: Determining malaria effects in rural Colombia. Soc Sci Med 1993, 37:1109-1114.

36. Vlassoff C, Bonilla E: Gender-related differences in the impact of tropical diseases on women: what do we know? J Biosoc Sci 1994, 26:37-53.

37. Tagny $C T$, Owusu-Ofori $S$, Mbanya D, Deneys V: The blood donor in subSaharan Africa: a review. Transfus Med 2010, 20:1-10.

38. Rajab JA, Waithaka PM, Orinda DA, Scott CS: Analysis of cost and effectiveness of pre-transfusion screening of donor blood and antimalarial prophylaxis for recipients. East Afr Med J 2005, 82:565-571.

39. Allain JP: Malaria and transfusion: a neglected subject coming back to the forefront. Clin Infect Dis 2010, 51:1199-1200.

40. Makler MT, Hinrichs DJ: Measurement of the lactate dehydrogenase activity of Plasmodium falciparum as an assessment of parasitemia. Am J Trop Med Hyg 1993, 48:205-210.

doi:10.1186/1475-2875-12-279

Cite this article as: Atchade et al:: Is a Plasmodium lactate

dehydrogenase (pLDH) enzyme-linked immunosorbent (ELISA)-based assay a valid tool for detecting risky malaria blood donations in Africa?. Malaria Journal 2013 12:279. 